\title{
Three-dimensional radar imaging of atmospheric layer and turbulence structures using multiple receivers and multiple frequencies
}

\author{
J.-S. Chen ${ }^{1}$, J. Furumoto ${ }^{2}$, and M. Yamamoto ${ }^{2}$ \\ ${ }^{1}$ Department of information and Network Communications, Chienkuo Technology University, Changhua, Taiwan \\ ${ }^{2}$ Research Institute for Sustainable Humanosphere, Kyoto University, Uji, Japan
}

Correspondence to: J.-S. Chen (jschen@ctu.edu.tw)

Received: 9 October 2013 - Revised: 1 April 2014 - Accepted: 2 April 2014 - Published: 5 August 2014

\begin{abstract}
The pulsed, beamwidth-limited atmospheric radar suffers from a finite resolution volume, making it difficult to resolve the small-scale irregularity structure of refractive index (or clear-air turbulence) in the scattering region. Multireceiver and multi-frequency imaging techniques were thus proposed to improve the spatial resolution of the measurements in the finite resolution volume. The middle and upper atmosphere radar (MUR; $34.85^{\circ} \mathrm{N}, 136.10^{\circ} \mathrm{N}$ ) possesses the capabilities of 5 frequencies, ranging from $46 \mathrm{MHz}$ to $47 \mathrm{MHz}$, and up to 25 receivers to carry out the imaging techniques. In this paper, we exhibit the three-dimensional (3-D) radar imaging utilizing five frequencies and 19 receivers of the MUR. The Capon method was employed for the process of imaging, and examinations of a wavy layer and turbulent structures were made, in which the spatial weighting effect on the imaging were mitigated beforehand. Information such as echo center and structure morphology in the resolution volume was then extracted. For example, the location distribution of echo centers could imply the traveling orientation of the wavy layer, which was correspondent with horizontal wind direction. Such information of wavy layer structure was more difficult to disclose without removal of the spatial weighting effect. This paper demonstrates an advanced application of 3-D radar imaging to some practical atmospheric phenomena.
\end{abstract}

Keywords. Meteorology and atmospheric dynamics (turbulence) - radio science (interferometry; signal processing)

\section{Introduction}

Three-dimensional (3-D) radar imaging using multi-receiver and multi-frequency is an advanced technique implemented in the UHF and VHF atmospheric radars to reconstruct the 3-D structures of refractivity irregularities in the atmosphere (i.e., clear-air turbulence), providing a spatial resolution at meter scale for the irregularity structures in the resolution volume (or the radar volume) (Yu and Palmer, 2001). There have been several VHF atmospheric radars in the world that can achieve multi-receiver and multi-frequency operations simultaneously, for example, the middle and upper atmosphere radar (MUR) (Hassenpflug et al., 2008), the OSWIN VHF radar (Chen and Zecha, 2009), the Chung-Li VHF radar (Chen et al., 2009), and so on. The latter two, however, have not made a complete operation/analysis of 3-D radar imaging yet. Recently, the Middle Atmosphere Alomar Radar SYstem (MAARSY) in Norway is one more VHF atmospheric radar which will fulfill multi-receiver and multi-frequency operations in the near future (Latteck et al., 2012). Among the plentiful studies of radar imaging, Hassenpflug et al. (2008) demonstrated the first use of operational 3-D radar imaging with five frequencies and 19 receivers of the MUR, and illustrated full 3-D views of a Kelvin-Helmholtz billow structure.

Mostly, the radar echoes received by multi-receiver and multi-frequency were treated separately for 2-D angular structure and for 1-D beam-direction structure, given the terminologies of coherent radar imaging (CRI) (Woodman, 1997; Palmer et al., 1998) and range imaging (RIM) (Palmer et al., 1999) or frequency-domain interferometric imaging (FII) (Luce et al., 2001). With CRI and/or RIM, there have been plenty of applications to the atmosphere, such as 
scattering mechanisms and dynamics in polar mesosphere summer echoes (PMSE) (Yu et al., 2001), small-scale variability of precipitation (Palmer et al., 2006), mitigation of bird contamination (Chen et al., 2007), effect of KelvinHelmholtz instability (KHI) on mean vertical wind (Chen et al., 2008a), KHI triggered by inertia-gravity wave (Luce et al., 2008), imaging of equatorial spread F (Chau et al., 2008), clutter suppression (Yu et al., 2010), measurement of aspect sensitivity of refractivity irregularities (Chen and Furumoto, 2013), applications to UHF radar (Chilson et al., 2003) and mobile weather radar (Isom et al., 2013), derivation of horizontal wind velocities (Sureshbabu et al., 2013), and so on.

In the processes of CRI and RIM, however, it is known that the radar beam (or antenna pattern) and range-weighting functions of the radar system have spatial weighting effect on the radar echoes. It is thus essential to remove these weighting effects from the imaging results to yield a more applicable imaging map in some circumstances. Nevertheless, removal of these weighting effects using theoretical mathematic forms may sometimes lead to an unrealistic aspect near the edge of the imaging map. To overcome such difficulty, the concept of adjustable weighting function was proposed for RIM (Chen and Zecha, 2009), CRI (Chen and Furumoto, 2011), and 3-D radar imaging (Chen et al., 2011). Based on the concept of adjustable weighting function and the associated analysis methods in the references, we fulfilled the 3-D imaging of some atmospheric structures to make an advanced application of multi-receiver and multi-frequency technique. In the literature, practical uses of 3-D radar imaging are not many. In view of this, more applications of the 3-D imaging technique to some specific atmosphere phenomena are worth carrying out.

Section 2 reviews the beam- and range-weighting effects on the radar imaging. Adaptable beam-weighting and rangeweighting functions are given briefly for the present study. Section 3 shows the 3-D imaging of a wavy layer as well as some small-scale variations of echoing structures in the radar volume. In Sect. 4, the atmospheric information extracted from the 3-D imaging structures is discussed. Conclusions are stated in Sect. 5.

\section{Observation and spatial weighting effect}

\subsection{Observations}

The radar echoes for 3-D radar imaging were collected by the MUR on 9-10 February, 2006. Only the data collected between 20:27-20:42 UT on 9 February (05:27-05:42 LT on 10 February), 2006, were used for the purpose of this case study. Figure 1 displays the array configuration of the MUR, where the full antenna array can be partitioned into 25 antenna groups for reception (i.e., the sub-arrays denoted from A1 to F5). In the experiment, the full antenna array was utilized for transmission in vertical, five equally spaced

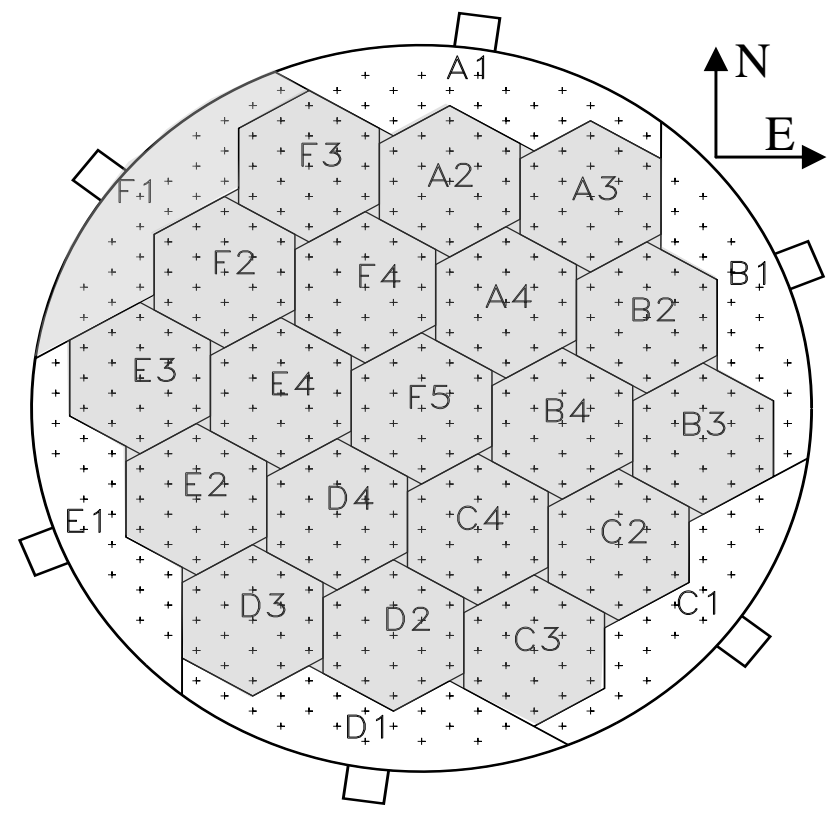

Figure 1. Array configuration of the MUR. Twenty-five antenna groups for transmission/reception are indicated from A1 to F5. The diameter of the array field is about $103 \mathrm{~m}$. The highlighted subarrays, as well as the full array, are employed for reception in the experiment, but the data of F1 subarray are not used in the study.

frequencies at $46.00,46.25,46.50,46.75,47.00 \mathrm{MHz}$ were transmitted sequentially during each pulse, and 21 antenna groups (i.e., receiving channels) were functioned to collect the data of each frequency, respectively. The antenna groups for reception are highlighted in Fig. 1, except for the full array which was also one of the 21 receiving channels. Interpulse period (IPP) and pulse length were 400 and $1 \mu$ s, respectively. The number of coherent integrations was 128 , providing a data time resolution of $0.256 \mathrm{~s}$ for each transmitter frequency. Sampling time step was $1 \mu$ s, giving a range step of $150 \mathrm{~m}$. The sampling range was between 3 and $22.2 \mathrm{~km}$.

In the process of RIM, the echoes collected from the full antenna array were used. In the process of CRI, the 19 receiving channels excluding the full array and $\mathrm{F} 1$ group were employed (termed as $\mathrm{Tx}_{\mathrm{full}} / \mathrm{Rx}_{1}$ mode hereafter), which were also used with the multi-frequency data for the 3-D radar imaging. Every 32 data points were used for an estimate of cross-correlation function, giving a time resolution of about $8 \mathrm{~s}$. The Capon method was employed for 3-D radar imaging. Readers can refer to the appendix for the basic algorithm of 3 -D radar imaging. More description of the radar capability can be found in Hassenpflug et al. (2008). 


\subsection{Equations for beam-weighting effect}

In the present experiment, the full antenna array was used for transmission, and the echoes collected from 19 receivers were applied to CRI. As proposed by Chen and Furumoto (2011), the effective beam-weighting function (BWF) suitable for this operational mode $\left(\mathrm{Tx}_{\mathrm{full}} / \mathrm{Rx}_{1}\right)$ can be expressed as

$W^{2}(\theta)=\exp \left(-\frac{\theta^{2}}{2 \theta_{e}^{2}}\right)$,

$\theta_{e}=\frac{c_{3} \theta_{e o}^{2}+c_{4}}{\mathrm{SNR}+10}+\theta_{e o}$,

$\theta_{e o}=c_{1}|\theta|^{3}+c_{2}$

where $W^{2}(\theta)$ is the two-way BWF, $\theta_{e}$ is the effective beamwidth, SNR is the signal-to-noise ratio of the echoes in $\mathrm{dB}$, and $\theta_{e o}$ indicates the effective beamwidth at infinite SNR. The variable $\theta$ is the angle with respect to the beam direction. The four coefficients, $\left(c_{1}, c_{2}, c_{3}\right.$, $\left.c_{4}\right)=(0.0096,3.1803,1.4751,-9.7430)$, were obtained from fitting processes with the echoes collected by Chen and Furumoto (2011) for the operational mode $\mathrm{Tx}_{\mathrm{full}} / \mathrm{Rx}_{1}$. Equations (2)-(3) are empirical, which contain the factors of SNR and $\theta$, and may have different forms for various operational modes of the MUR as well as other radar systems. In addition, Eq. (2) is valid for $\mathrm{SNR}>-10 \mathrm{~dB}$ to give a positive value of $\theta_{e}$

\subsection{Equations for range-weighting effect}

The range-weighting function (RWF) is a convolution result between pulse envelope and system impulse response, in which the system impulse response is the inverse Fourier transform of the receiver filter function. Although a theoretical Gaussian RWF is commonly assumed for a rectangular pulse shape with its matched filter (Franke, 1990), some studies have proposed various shapes of RWF for different combinations of pulse shapes and receiver filter functions in the practical process of RIM (Chen and Zecha, 2009). Chen and Zecha (2009) also contributed the concept of adaptable RWF for improving the continuity of the imaged powers (or brightness) around the boundaries of the sampling gates; their RWF is adaptive to SNR. A more recent study made by Chen et al. (2014) further proposed an improved RWF that is adaptive to SNR and range within the sampling gate; these RWFs could be useful for some practical data analysis, for example, extending the RIM process to a larger range extent for 3-D radar imaging in a sampling gate. To have suitable expressions of adaptable RWF for use of the present study, we reexamined the MUR data demonstrated by Chen et al. (2014) that were oversampled but employed the same pulse envelope and receiver filter function with the present experiment. A set of equations for RWF were obtained as follows:
$W^{2}(r) \cong \exp \left(-\frac{r^{2}}{2 \sigma_{r}^{2}}\right)=\exp \left(-\frac{r^{2}}{\sigma_{z}^{2}}\right)$,

$\sigma_{z}=\frac{a_{3} \sigma_{z o}^{3}+a_{2} \sigma_{z o}^{2}+a_{1} \sigma_{z o}+a_{o}}{(\mathrm{SNR}+10)^{1.2}}+\sigma_{z o}$,

$\sigma_{z o}=b_{1}|r|^{3}+b_{2}$,

where $W^{2}(r)$ is the two-way RWF, $\sigma_{r}$ is the standard deviation of the Gaussian RWF, and $\sigma_{z}$ is defined as the effective standard deviation of $W^{2}(r)$ in this paper. SNR is in dB. $\sigma_{z o}$ indicates the effective standard deviation of $W^{2}(r)$ at infinite SNR. The variable $r$ is the range with respect to the range center of the sampling gate. The coefficients, $\left(a_{0}, a_{1}\right.$, $\left.a_{2}, a_{3}\right)=(-10742.923,312.5499,-2.7278,0.0080723)$ and $\left(b_{1}, b_{2}\right)=\left(1.1457 \times 10^{-5}, 73.4538\right)$, were obtained from fitting processes with the MUR data used in Chen et al. (2014). Note again, Eqs. (5)-(6) are empirical, which are adaptive to SNR and $r$, and may have different forms for various radar parameters as well as other radar systems. In addition, Eq. (5) is valid for SNR $>-10 \mathrm{~dB}$. Equations (4)-(6) have the similar forms to Equations (1)-(3).

Figure 2 demonstrates the effect of range- and SNRdependent RWF on a RIM case. For a discussion with wind field later, the time starts from the right side of the abscissa. In the panel a, the RIM was executed for each sampling gate within the range extent of -75 to $75 \mathrm{~m}$. The range step of imaging was $1 \mathrm{~m}$ and the time step was about $16 \mathrm{~s}$. Equations (5) and (6) were employed for correcting the RWF effect of individual sampling gate. As seen, a wavy layer appeared in the sampling gate centered at $5.325 \mathrm{~km}$. The panel b is the RIM with the commonly defined RWF (i.e., $\sigma_{z}=75 \mathrm{~m}$ or $\sigma_{r} \approx 53 \mathrm{~m}$ in Eq. 4) in correction of the imaged power. As seen, the discontinuity or over correction of the imaged power at gate boundaries is evident as compared with the panel a. If the RWF effect is not corrected, the absence of the imaged power occurs very often at gate boundaries, as observed in the panel c. Accordingly, we can adopt the rangeand SNR-dependent RWF for the present case.

In theory, the imaging process can be extended to the location outside the pulse-defined range extent, in case the RWF effect can be compensated thoroughly. In practice, it may not be workable to extend the imaging process too far from the range center. The panels $\mathrm{d}-\mathrm{f}$ examined this issue in more detail with the wavy layer in the sampling gate centered at $5.325 \mathrm{~km}$. In the panel d, the RIM of the wavy layer was performed between -150 and $150 \mathrm{~m}$ only for the sampling gate centered at $5.325 \mathrm{~km}$, and the SNR- and range-dependent RWF was employed. As a result, the obtained wavy layer structure was very close to that in the panel a. By contrast, the RIM with the commonly defined RWF (i.e., $\sigma_{z}=75 \mathrm{~m}$ or $\sigma_{r} \approx 53 \mathrm{~m}$ in Eq. 4) in correction of the imaged power is displayed in the panel e, in which over correction of the imaged 
(a) Six-gate RIM: $\sigma_{z}$ adaptive to SNR and range

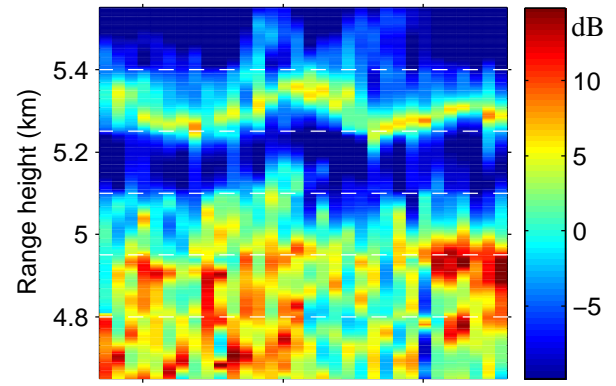

(b) Six-gate RIM: $\sigma_{\mathrm{z}}=75 \mathrm{~m}$

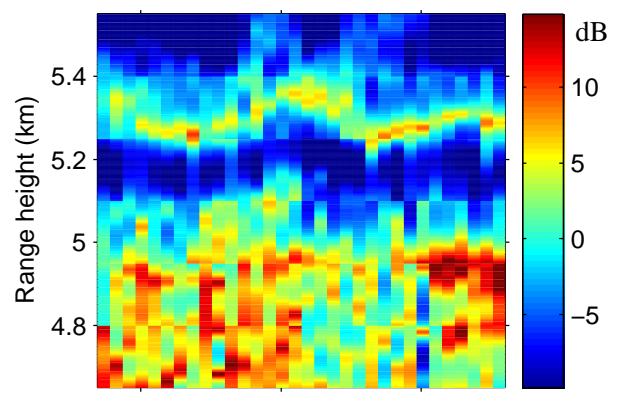

(c) Six-gate RIM without correction

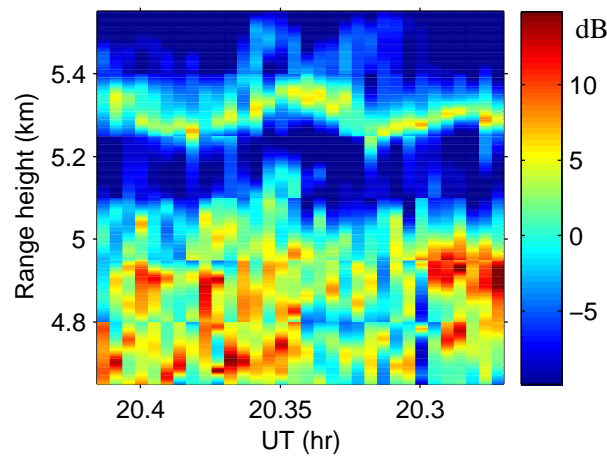

(d) One-gate RIM: $\sigma_{z}$ adaptive to SNR and range

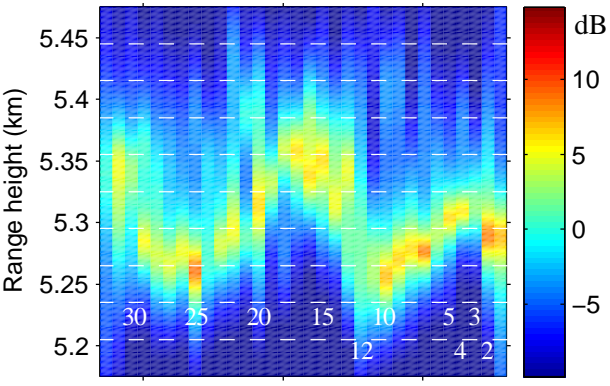

(e) One-gate RIM: $\sigma_{\mathrm{z}}=75 \mathrm{~m}$

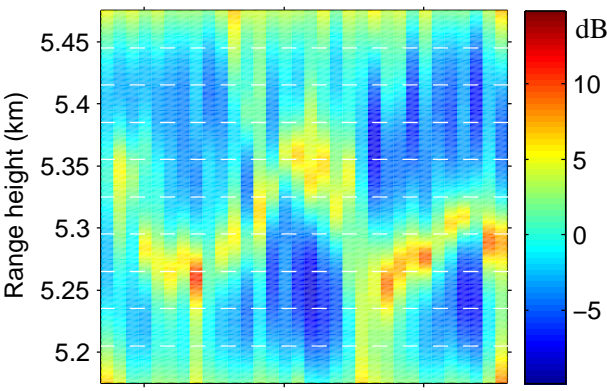

(f) One-gate RIM: $\sigma_{z}$ adaptive to SNR

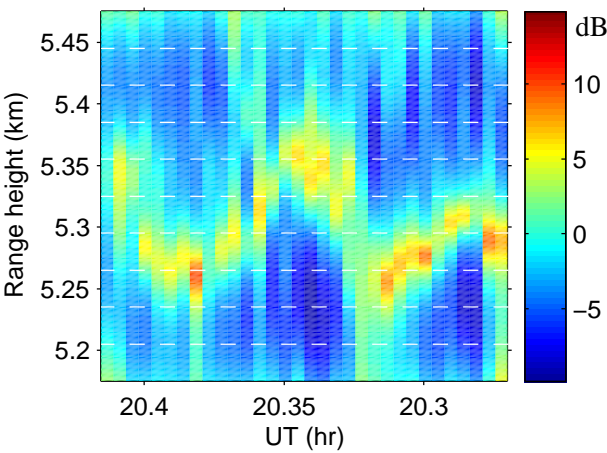

Figure 2. Range imaging of a wavy layer, observed by the MUR. The range step of imaging is $1 \mathrm{~m}$, and time resolution is about $16 \mathrm{~s}$. The time starts from the right side of the abscissa. (a) Range imaging for six sampling gates with range centers at 4.725, 4.875, 5.025, 5.175, 5.325 , and $5.475 \mathrm{~km}$. Imaging process of each gate is from -75 to $75 \mathrm{~m}$ with respect to the range center of the sampling gate, and SNR- and range-dependent RWF is used in correction of brightness value. (b) and (c) are similar to (a) but with a commonly defined RWF in (b) and without correction of RWF in (c). The three panels of (d), (e) and (f) are the imaging results for the sampling gate centered at $5.325 \mathrm{~km}$, but the imaging process is from -150 to $150 \mathrm{~m}$ with respect to the range center of the sampling gate and uses different RWFs in correction of brightness value. The numbers given in the panel (d) denote the time slots.

power can be seen around the upper and lower edges of the imaging map. Such an over correction of the imaged power can be mitigated but still visible when the SNR-dependent RWF was employed, as shown in the panel f. For producing the panel $\mathrm{f}$, we have used the following form for the SNRdependent RWF:

$\sigma_{z}=\frac{1}{\frac{a}{(\mathrm{SNR}+10)^{C}}+b}-d$,

where the coefficients, $(a, b, c, d)=(-0.025073,0.024557$, $0.2,0$ ), were obtained by fitting the present radar data with the computing process suggested by Chen and Zecha (2009). Note that SNR should be larger than $-10 \mathrm{~dB}$ to give a nonnegative value of $\sigma_{z}$.

In view of the comparison in Fig. 2, the RWF adaptive to SNR and range is more suitable for extending the imaging to the locations outside the pulse-defined range extent; it can avoid sudden change of the brightness value at gate boundaries and provide a smoother imaging structure through the coverage of a sampling gate. Nevertheless, it should be notified here that the SNR- and range-dependent RWF is not a must for all circumstances. Usually, the SNR-dependent 
RWF proposed by Chen and Zecha (2009) or Chen et al. (2009) is enough for a normal RIM within the pulsedefined range extent (i.e., -75 to $75 \mathrm{~m}$ for the present experiment).

\section{Three-dimensional structure of a wavy layer}

For the wavy layer shown in Fig. 2d, the 3-D structures at various time slots were examined. Some typical results are shown in Figs. 3 and 5. For clarity of inspection, the contoured angular brightness distributions of nine slices at range locations of $-120,-90,-60,-30,0$ (range center), 30, 60, 90 , and $120 \mathrm{~m}$ are displayed. The unit of brightness value was $\mathrm{dB}$, but normalization was made for individual time slots. The thick dashed curve in black links the major echo centers at several range locations in the contour plot for convenience of inspection.

In Figs. 3 and 5, two kinds of RIM are also displayed on the range-meridional plane: one is the sum of the brightness values at equal-range surface (termed as summed-RIM, represented with black solid curve), which is analogous to the traditional 1-D RIM; the other is the profile of the brightness values at zenith direction (termed as zenith RIM, indicated by red solid curve). Since the RIM can reveal the layer structure along the range direction, the zenith RIM indicates a thinner layer structure than the summed RIM although the difference between the two RIM profiles is not very large. This can be attributed to the function that the zenith RIM rejects the echoes at off-zenith directions. The benefit of zenith RIM in 3-D radar imaging has been proposed by Hassenpflug et al. (2008) and Yu et al. (2010), for example, suppressing the aircraft echoes at off-zenith angles to produce the layer structures with less interference. However, Hassenpflug et al. (2008) also pointed out that the zenith RIM from 3-D radar imaging has lower resolution than 1-D RIM (i.e., retrieved solely from the multi-frequency data collected by the full-array receiving channel and with a vertical radar beam).

In Fig. 3, the time slots 4, 3, and 2 indicated in Fig. 2d are displayed, which were around a hump of the wavy layer. Notice that the main body of the wavy layer at the three time slots was located below the range center (see Fig. 2). Panel a of Fig. 3 shows the imaging structures without correction of spatial weighting effect (termed as original imaging hereafter) at the three time slots, and the panel $b$ gives the imaging structures after correction of spatial weighting effect (termed as modified imaging hereafter). As observed at the time slot 3, two echo centers can be revealed clearly below the range location of $30 \mathrm{~m}$ in the modified imaging; by contrast, the two echo centers are not obvious in the original imaging. On the other hand, the time slots 2 and 4 had the echo centers at opposite zonal directions, which can be disclosed more clearly in the modified imaging. Such features can be explained with the schematic plot of a wavy layer in Fig. 4 , in which the time instants $t_{2}, t_{3}$, and $t_{4}$ correspond to the time slots 2, 3, and 4 indicated in Fig. 2d, respectively. At the time instant $t_{3}$, the echoes may come from both sides of the crest, giving two echo centers at both sides of the zenith in the imaging map. At the time instants $t_{2}$ or $t_{4}$, the echoes return mainly from the right or left side of the crest, giving the echo center at positive or negative zenith angle.

More examinations have demonstrated that the 3-D radar imaging can disclose the variation of echo center with range location in the sampling gate. Two more situations at the time slots 12 and 20 are shown in Fig. 5. At time slot 12, the main echo center was around the range location of $-45 \mathrm{~m}$ and from positive zonal direction. With increase of range, the echo center shifted to negative zonal direction. Such a variation of echo center with range location can be figured out according to the radar beam location at time instant $t_{12}$ in Fig. 4. As illustrated, the body of the wavy layer is very slanted in the illumination volume. The 3-D radar imaging can retrieve such slanted structure in the illumination volume, yielding the imaging map at time slot 12 . By contrast, the body of the wavy layer at time instant $t_{20}$ shows an opposite slope to that at time instant $t_{12}$, giving the imaging map exhibited in Fig. 5.

\section{Discussion}

In the previous section, we illustrate the capability of 3-D radar imaging for the MUR. Further applications of the imaging results are demonstrated in this section.

\subsection{Case 1}

The first case is the wavy layer shown in Fig. 2 d. The angular locations of echo centers estimated at nine range locations (i.e., at the nine slices in Fig. 3), are displayed in Fig. 6 for the whole time period, where the panel a shows the echo centers estimated from the original imaging, and the panel $b$ displays the echo centers obtained from the modified imaging. The method for determination of the locations of echo centers is the contour-based approach proposed by Chen et al. (2008b).

The echo centers estimated from the modified imaging are supposed to be applicable if the spatial weighting effect has been removed properly. Nevertheless, in Fig. 6 we discarded the echo centers with brightness value lower than $30 \%$ of the maximum brightness level for raising the reliability of echo center distribution. Moreover, the situations of single and multiple echo centers were displayed separately for a more detailed inspection. In panel a of Fig. 6, the echo centers clustered around zenith, and most of them were the situation of single center (see the middle and right-hand plots). After correction of spatial weighting effect, however, multicenter situation was disclosed to be plentiful, as seen in the right-hand plot of the panel $b$. In addition, we can observe a clear northeast-southwest arrangement of echo center locations from the multi-center locations. 
(a) Original
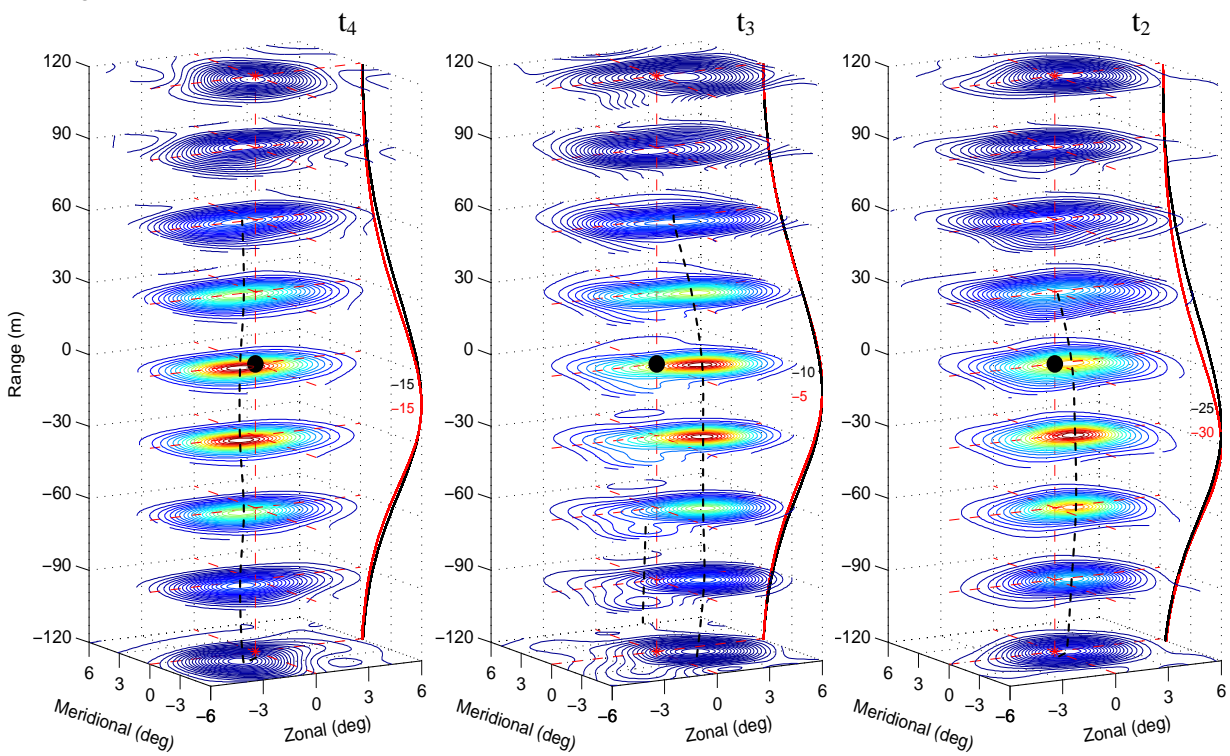

(b) Modified

$\mathrm{t}_{4}$

$t_{3}$
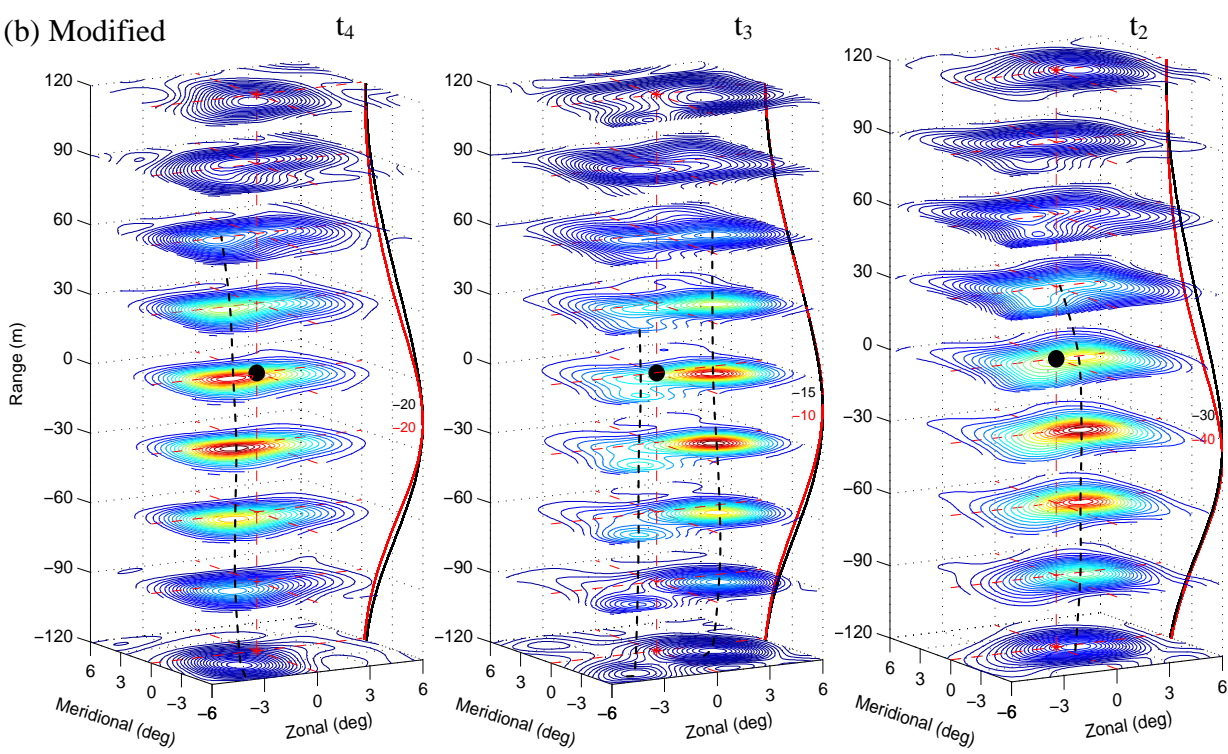

Figure 3. 3-D radar imaging at the time slots 4, 3, and 2 (from left to right) indicated in Fig. 2d. The 2-D contoured brightness distributions of nine slices at range locations of $-120,-90,-60,-30,0,30,60,90$, and $120 \mathrm{~m}$ are displayed. The $0 \mathrm{~m}$ location corresponds to the range of $5.325 \mathrm{~km}$. The unit of the brightness value is $\mathrm{dB}$, but normalization is made for individual time slot. (a) and (b) are the imaging results, respectively, with and without correction of spatial weighting effect. The thick dashed curve in black links the major echo centers at several range locations. Black spot is the central location of the radar volume. Two types of 1-D RIM profiles are shown on the range-meridional plane: (black) summation of the brightness values at equal-range surface, (red) the brightness values along the zenith direction.

The northeast-southwest arrangement of echo center locations can be explained with the morphology of the wavy layer sketched in Fig. 4, assuming the wavy layer travels with a sheared horizontal wind in the northeast direction and the wave shape is slanted to the leeward due to the wind-shear effect. In such circumstance, the wavefront of the wavy layer is in the northwest-southeast direction. For this slanted wavy layer, the radar echoes return mostly from either left or right sides of the crest, leading to the location distribution of echo centers in northeast-southwest arrangement. Moreover, the radar echoes prefer coming from the upstream side of the crest (Chen et al., 2008a), that is, the southwest direction for the present case. This is verified by the values of $\mathrm{Zd}$ and $\mathrm{Md}$ given in each plot. $\mathrm{Zd}$ (or Md) is the difference between the numbers of positive angles and negative angles in zonal (or meridional) direction, presented as a percentage of the total 


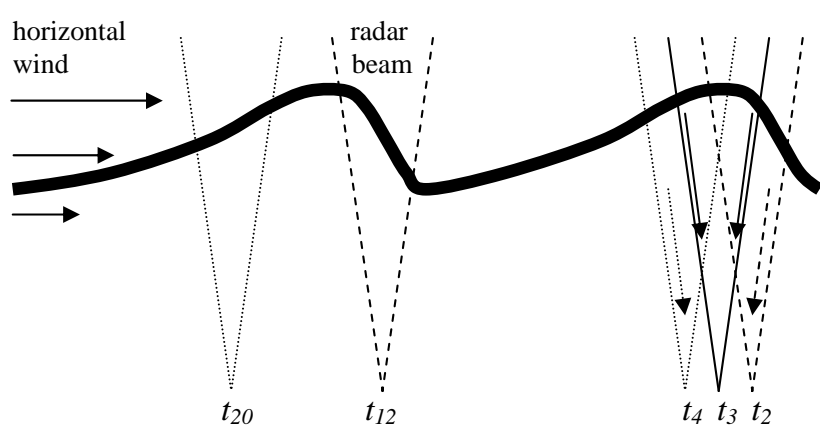

Figure 4. A wavy layer structure in a wind-sheared atmosphere. Radar beam is vertical. $t_{n}$ denotes different observational times corresponding to the time slots indicated in Fig. $2 d$.
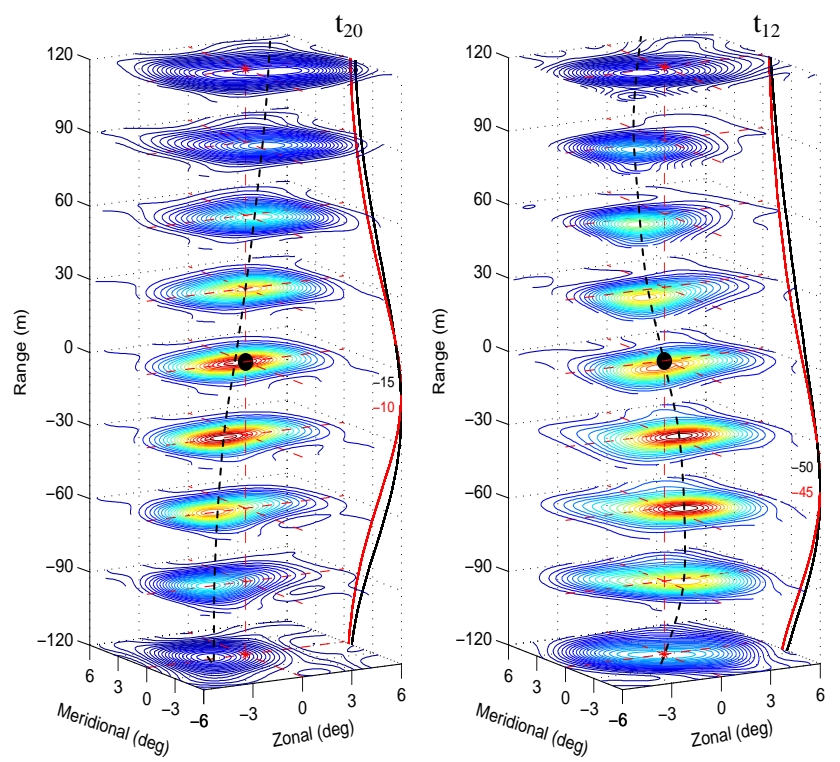

Figure 5. Same as Fig. 3, but showing the imaging results at the time slots 20 and 12 indicated in Fig. 2d. Mitigation of spatial weighting effect is made.

number in each plot. Referring to the left-hand plot including single- and multi-center situations, both values of $\mathrm{Zd}$ and Md were below zero apparently no matter in the original or modified 3-D imaging; this implies more echo centers in the lower-left part of the plot and reveals the scenario sketched in Fig. 4.

To validate the above inference, wind field was examined. The upper panel of Fig. 7 shows the mean Doppler velocity of 64-point spectrum from the full-array receiving channel. Such obtained Doppler velocity is a combination of vertical and horizontal winds although the transmitted radar beam is vertical. It is noticed that the time variation in Doppler velocity between 4.8 and $5.4 \mathrm{~km}$ was consistent with the wavy layer pattern, that is, the Doppler velocity was downward (upward) on the upstream (downstream) side of the crest (refer to Fig. 4 for the schematic plot of the wavy layer). Since (a) from original 3-D imaging
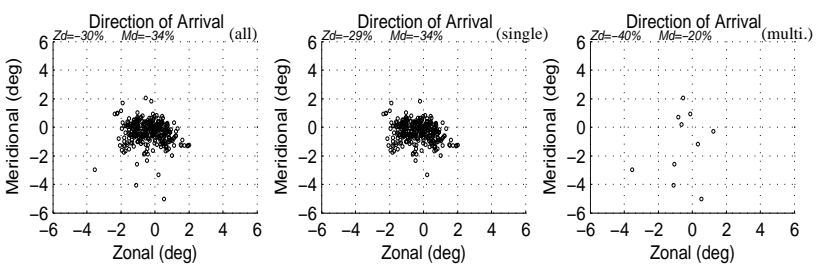

(b) from modified 3-D imaging
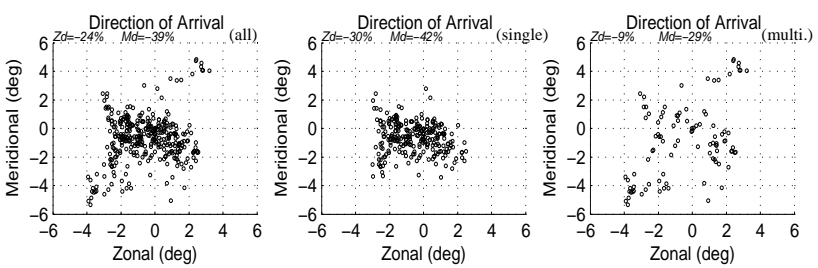

Figure 6. Angular locations of echo centers at range locations of $-120,-90,-60,-30,0,30,60,90$, and $120 \mathrm{~m}$ in the sampling gate centered at $5.325 \mathrm{~km}$. (a) and (b) result from the 3-D radar imaging of the wavy layer structure with and without correction of spatial weighting effect, respectively. The left-hand plots show all estimates, and the middle and right-hand plots display the situations of single center and multiple centers, respectively.

the radar echoes were returned mainly from both sides of the crest due to the tilt structure of the wavy layer, the velocity component projecting from horizontal movement of echoing regions could be the major part of the Doppler velocity for the vertical beam. For example, the Doppler velocity is $\sim 0.35 \mathrm{~m} \mathrm{~s}^{-1}$ when the radar target is located at $2^{\circ}$ off zenith and travels horizontally at a speed of $10 \mathrm{~m} \mathrm{~s}^{-1}$. This value of Doppler velocity is comparable to that shown here. The horizontal wind with a speed of $10 \mathrm{~m} \mathrm{~s}^{-1}$ is common in the atmosphere, as shown in the panel b of Fig. 7. To estimate horizontal wind, the full correlation analysis (FCA) of spaced antenna (Briggs, 1984) was employed with the data sets of subarrays F5, A4, and B4. To obtain more reliable wind values from FCA, 512 data points were used, resulting in a time step of about $4.5 \mathrm{~min}$. As seen in the panel b, the horizontal wind blew toward the northeast direction approximately, which could propel the wavy layer structure to the northeast-southwest direction. Accordingly, the specific location distribution of echo centers in Fig. 6b makes sense indeed. Note also the wind shear occurring in the range interval between 5.2 and $5.4 \mathrm{~km}$, which can deform the wavy layer into that sketched in Fig. 4.

It is worth an examination if the above achievement of 3D radar imaging can be acquired from 2-D radar imaging (CRI) or not. This is illustrated in Fig. 8, where the echo centers obtained from the CRI are shown. Except for the case of single center that was in small number, the locations of echo centers were more scattered than those of 3-D radar imaging, even the BWF effect has been mitigated for the CRI (the panel b). Moreover, the values of $\mathrm{Zd}$ and $\mathrm{Md}$ in the left-hand 

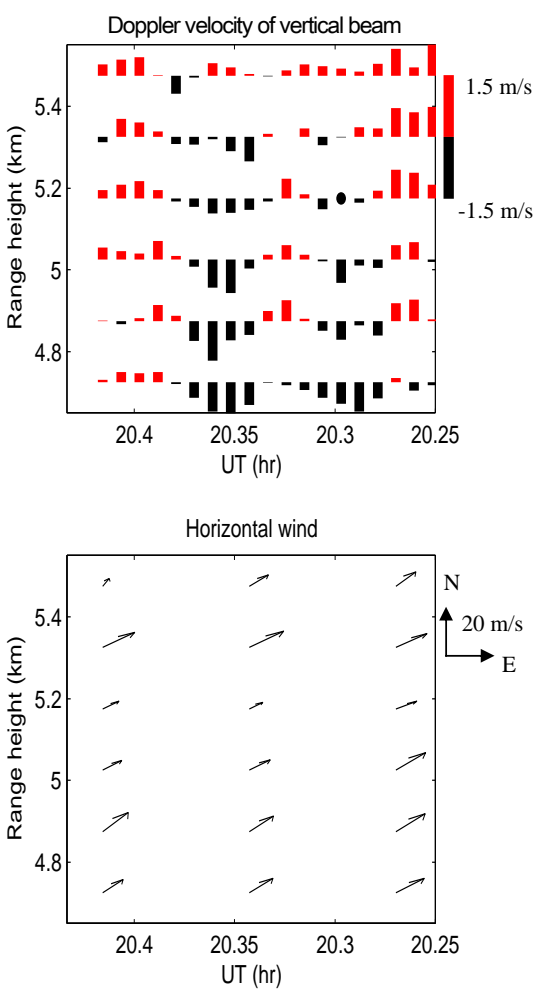

Figure 7. (Upper) mean Doppler velocity estimated from the Doppler spectrum of 64 data points of the vertical beam, and (lower) horizontal wind derived from full-correlation analysis of spaced antenna with 512 data points.

two plots were closer to zero as compared with Fig. 6. In view of these features, the CRI did not disclose the orientation of the wavy layer as clearly as the 3-D radar imaging. In addition to the above consequence, we can also observe in the CRI that multiple-center situation is more plentiful than the single-center situation, which contrasted with the result of 3$\mathrm{D}$ radar imaging; this is interpretable. The CRI is a composite result along the range direction, and so the echoes at different range locations are superimposed. In case more than one scattering center are located at different directions and range locations, there may be several echo centers in the 2-D angular brightness distribution. In view of this, the benefit of 3-D radar imaging with correction of spatial weighting effects is evident, for this case at least.

\subsection{Case 2}

Case 1 is a definite wavy-layer structure. In Fig. 2a, we can observe different turbulent structures below $\sim 5 \mathrm{~km}$, which are contrasting with the wavy layer structure. We examined the echoes in the sampling gate between 4.8 and $4.95 \mathrm{~km}$ for comparison with the first case. The location distribution of echo centers from 3-D radar imaging, like that in Fig. 6, are shown in Fig. 9. Compared with the case of wavy layer, the echo centers in Fig. 9 were more scattered in both original (a) from original 2-D imaging
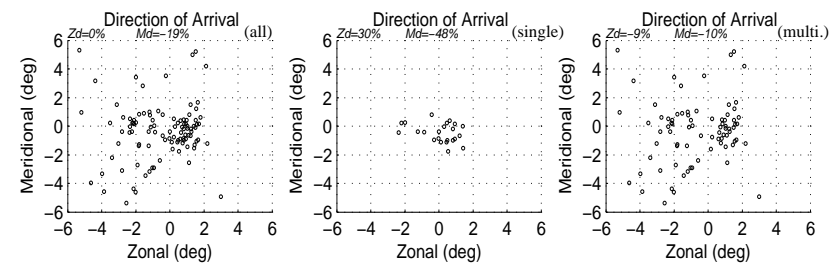

(b) from modified 2-D imaging
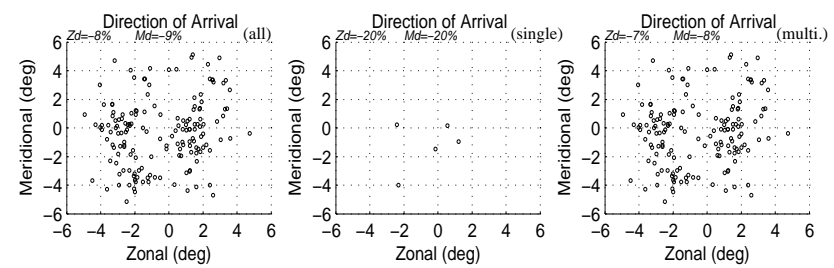

Figure 8. Similar to Fig. 6, but resulting from the 2-D radar imaging of multi-receiver echoes.

(a) from original 3-D imaging

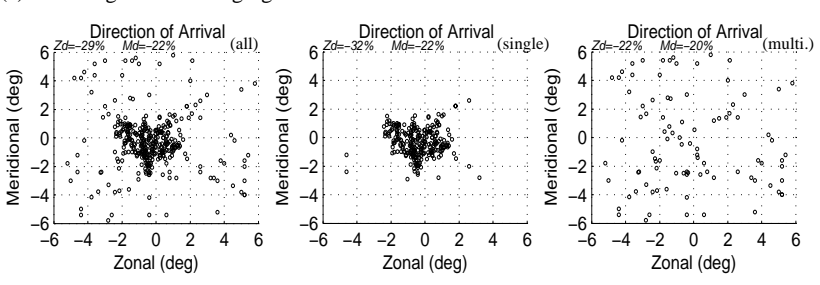

(b) from modified 3-D imaging

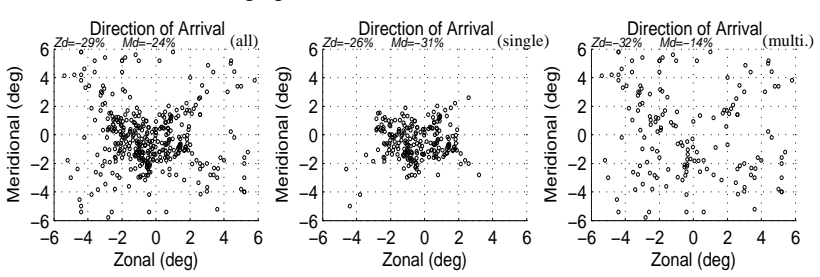

Figure 9. Similar to Fig. 6, but obtained from the echoes in the sampling gate between 4.8 and $4.95 \mathrm{~km}$.

and modified imaging results, which was mainly due to the scattering distribution of multiple centers shown in the righthand plots. Such feature seems in consistent with the spatial characteristics of turbulent structures. More noticeable information is that the values of $\mathrm{Zd}$ and $\mathrm{Md}$ were negative and comparable to those in Fig. 6, also indicating a favorite direction of southwest for the radar echoes.

\section{Conclusions}

Three-dimensional (3-D) radar imaging using multi-receiver and multi-frequency has been applied to retrieve the atmospheric structure within the radar volume. The observation was executed with the middle and upper atmospheric radar (MUR). We have shown the variations of echo centers in the 
radar volume, verifying the capability of 3-D radar imaging in providing high-resolution structure.

To make a more sophisticated study with 3-D radar imaging, our examinations have considered the beam- and rangeweighting effects on the radar echoes. Several equations adaptive to off-beam direction, SNR, and range location were used to mitigate the spatial weighting effect for the present radar system and experimental mode. We have demonstrated the benefit of mitigating the spatial weighting effect for a wavy layer structure. For example, the location distribution of echo centers in the radar volume could indicate the traveling direction of the wavy layer structure. Such feature of 3-D imaging structure was found to be associated closely with wind field. In comparison, the above feature of 3-D imaging structure was more difficult to disclose without mitigation of spatial weighting effect, and it could not be found easily from the 2-D radar imaging (CRI) with only multi-receiver echoes.
An examination of turbulent structures was also made with the 3-D imaging technique. Compared with the case of wavy layer, the estimated echo centers were more scattered, conforming with the spatial characteristics of turbulent structures. In addition, in both cases the upstream side of the radar beam (i.e., the southwest direction) was the favorite incident direction of the radar echoes, which is possibly due to the slanted layer/turbulence structures caused by wind shear effect.

The two cases shown in this paper have exhibited some potential applications of 3-D radar imaging implemented in the VHF atmospheric radar. In view of rare publications of 3 $\mathrm{D}$ radar imaging in the atmospheric radar community, more studies with the benefit of multi-receiver and multi-frequency technique are worth carrying out in the future. 


\section{Appendix A: Equations of 3-D radar imaging}

An original and more detailed description of 3-D radar imaging for the atmosphere can refer to Yu and Palmer (2001), and a simplified introduction was given by Chen et al. (2011). The following equations are extracted from Chen et al. (2011).

Given $N$ spatially separated receivers at locations of $\boldsymbol{D}_{1}$, $\boldsymbol{D}_{2}, \ldots, \boldsymbol{D}_{n}$ and transmitting $M$ carrier frequencies at $f_{1}$, $f_{2}, \ldots, f_{M}$ sequentially during each pulse, the signals from these receivers and carrier frequencies can be expressed as a column vector:

$$
\begin{gathered}
\mathbf{S}(t)=\left[S_{11}(t) S_{12}(t) \ldots S_{1 N}(t)\right. \\
S_{21}(t) S_{22}(t) \ldots S_{2 N}(t) \\
\vdots \\
\left.S_{M 1}(t) S_{M 2}(t) \ldots S_{M N}(t)\right]^{\mathrm{T}}
\end{gathered}
$$

where the superscript $\mathrm{T}$ represents the transpose, and $S_{i j}(t)$ is the signal from carrier frequency $i$ and receiver $j$. The following cross-correlation function of the signal $\mathbf{S}(t)$ is required in the imaging process:

$\mathbf{V}(t)=\mathbf{S}(t) \mathbf{S}^{\mathrm{H}}(t)=\left[\begin{array}{cccc}\mathbf{V}_{11} & \mathbf{V}_{12} & \cdots & \mathbf{V}_{1 M} \\ \mathbf{V}_{21} & \mathbf{V}_{22} & \cdots & \mathbf{V}_{2 M} \\ \vdots & \vdots & \ddots & \vdots \\ \mathbf{V}_{M 1} & \mathbf{V}_{M 2} & \cdots & \mathbf{V}_{M M}\end{array}\right]$,

where the superscript $\mathrm{H}$ represents the Hermitian (conjugate and transpose) operator. $\mathbf{V}(t)$ is a $M N \times M N$ matrix (termed as visibility matrix), and $\mathbf{V}_{p q}$ is a $N \times N$ matrix consisting of cross-correlation functions between receivers for the frequency pair of $p$ and $q$. For example,

$\mathbf{V}_{2 M}=\left[\begin{array}{cccc}S_{21} S_{M 1}^{*} & S_{21} S_{M 2}^{*} & \cdots & S_{21} S_{M N}^{*} \\ S_{22} S_{M 1}^{*} & S_{22} S_{M 2}^{*} & \cdots & S_{22} S_{M N}^{*} \\ \vdots & \vdots & \ddots & \cdots \\ S_{2 N} S_{M 1}^{*} & S_{2 N} S_{M 2}^{*} & \cdots & S_{2 N} S_{M N}^{*}\end{array}\right]$, where asterisk indicates the conjugate of a complex number. Note that the time variable $t$ has been omitted from Eq. (A3) for notational simplicity. $\mathbf{V}_{p q}$ is the estimate of visibility function without time lag, that is, imaging for different Doppler frequencies of the signals is not made.

The imaging process is to retrieve the so-called brightness distribution via the visibility matrix $\mathbf{V}$, defined as

$B(\boldsymbol{a}, R)=\frac{1}{W^{2}(\boldsymbol{a}, R)} \mathbf{w}^{\mathrm{H}} \mathbf{V w}$,

where $\boldsymbol{a}=[\sin \theta \sin \varphi, \sin \theta \cos \varphi, \cos \theta]$, which is the angular direction at zenithal angle $\theta$ and azimuthal angle $\varphi$. The variable $R$ is the range. $W(\boldsymbol{a}, R)$ is the spatial weighting function for the echoes in the radar volume, formed by radar beamand range-weighting functions. Matrix $\mathbf{w}$ is the weighting vector for retrieving the brightness distribution, given as

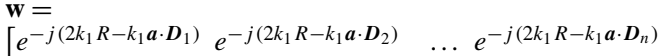

$$
\begin{aligned}
& e^{-j\left(2 k_{2} R-k_{2} \boldsymbol{a} \cdot \boldsymbol{D}_{1}\right)} e^{-j\left(2 k_{2} R-k_{2} \boldsymbol{a} \cdot \boldsymbol{D}_{2}\right)} \quad \ldots e^{-j\left(2 k_{2} R-k_{2} \boldsymbol{a} \cdot \boldsymbol{D}_{n}\right)} \\
& \begin{array}{cccc}
\vdots & \vdots & \ddots & \vdots \\
e^{-j\left(2 k_{M} R-k_{M} \boldsymbol{a} \cdot \boldsymbol{D}_{1}\right)} & e^{-j\left(2 k_{M} R-k_{M} \boldsymbol{a} \cdot \boldsymbol{D}_{2}\right)} & \ldots & \left.e^{-j\left(2 k_{M} R-k_{M} \boldsymbol{a} \cdot \boldsymbol{D}_{n}\right)}\right]^{\mathrm{T}}
\end{array}
\end{aligned}
$$

where $k_{i}$ is the wave number of carrier frequency $i$. Substituting Eq. (A5) into (A4) yields the so-called Fourier brightness that has a coarse resolution. To enhance the imaging resolution, the Capon method is one of the choices:

$B(\boldsymbol{a}, R)=\frac{1}{W^{2}(\boldsymbol{a}, R)} \frac{1}{\mathbf{e}^{\mathrm{H}} \mathbf{V}^{-1} \mathbf{e}}$,

where $\mathbf{e}$ has the same form as Eq. (A5). Equations (A4) and (A6) indicate that the brightness value is weighted by the spatial weighting function $W(\boldsymbol{a}, R)$. Removal of $W(\boldsymbol{a}, R)$ from the brightness value is thus necessary to improve the reconstruction of 3-D atmospheric structure. 
Acknowledgements. This work was supported by the National Science Council of ROC (Taiwan), grant no. NSC102-2111-M-270001 and MOST103-2111-M-270-001. The experiment was supported by the MUR International Collaborative Research Program (ref. no. 2005-O25), Research Institute for Sustainable Humanosphere, Kyoto University, Japan. We would like to thank Toshitaka Tsuda for encouraging the experiment with the MUR radar.

Topical Editor C. Jacobi thanks two anonymous referees for their help in evaluating this paper.

\section{References}

Chau, J. L., Hysell, D. L., Kuyeng, K. M., and Galindo, F. R.: Phase calibration approaches for radar interferometry and imaging configurations: equatorial spread F results, Ann. Geophys., 26, 2333-2343, doi:10.5194/angeo-26-2333-2008, 2008.

Chen, J.-S. and Furumoto, J.: A novel approach to mitigation of radar beam weighting effect on coherent radar imaging using VHF atmospheric radar, IEEE T. Geosci. Remote, 49, 30593070, doi:10.1109/TGRS.2011.2119374, 2011.

Chen, J.-S. and Furumoto, J.: Measurement of atmospheric aspect sensitivity using coherent radar imaging after mitigation of radar beam weighting effect, J. Atmos. Ocean. Tech., 30, 245-259, doi:10.1175/JTECH-D-12-00007.1, 2013.

Chen, J.-S. and Zecha, M.: Multiple-frequency range imaging using the OSWIN VHF radar: Phase calibration and first results, Radio Sci., 44, RS1010, doi:10.1029/2008RS003916, 2009.

Chen, J.-S., Hassenpflug, G., and Yamamoto, M.: Tilted refractiveindex layers possibly caused by Kelvin-Helmholtz instability and their effects on the mean vertical wind observed with multiple-receiver and multiple-frequency imaging techniques, Radio Sci., 43, RS4020, doi:10.1029/2007RS003816, 2008a.

Chen, J.-S., Hoffmann, P., Zecha, M., and Hsieh, C.-H.: Coherent radar imaging of mesosphere summer echoes: Influence of radar beam pattern and tilted structures on atmospheric echo center, Radio Sci., 43, RS1002, doi:10.1029/2006RS003593, 2008 b.

Chen, J.-S., Su, C.-L., and Chu, Y.-H., Hassenpflug, G., and Zecha, M.: Extended application of a novel phase calibration method of multiple-frequency range imaging to the Chung-Li and MU VHF radars, J. Atmos. Ocean. Tech., 26, 2488-2500, 2009.

Chen, J.-S., Chen, C.-H., and Furumoto, J.: Radar beam- and rangeweighting effects on three-dimensional radar imaging for the atmosphere, Radio Sci., 46, RS6014, doi:10.1029/2011RS004715, 2011.

Chen, J.-S., Su, C.-L., Chu, Y.-H., Kuong, R.-M., and Furumoto, $\mathrm{J} .:$ Measurement of range-weighting function for range imaging of VHF atmospheric radars using range oversampling technique, J. Atmos. Ocean. Tech., 31, 47-61, doi:10.1175/JTECH-D-12$00236.1,2014$.

Chen, M.-Y., Yu, T.-Y., Chu, Y.-H., Brown, W. O. J., and Cohn, S. A.: Application of Capon technique to mitigate bird contamination on a spaced antenna wind profiler, Radio Sci., 42, RS6005, doi:10.1029/2006RS003604, 2007.

Chilson, P. B., Yu, T.-Y., Strauch, R. G., Muschinski, A., and Palmer, R. D.: Implementation and validation of range imaging on a UHF radar wind profiler, J. Atmos. Ocean. Tech., 20, 987996, 2003.
Franke, S. J.: Pulse compression and frequency domain interferometry with a frequency-hopped MST radar, Radio Sci., 25, 565574, 1990.

Hassenpflug, G., Yamamoto, M., Luce, H., and Fukao, S.: Description and demonstration of the new Middle and Upper atmosphere Radar imaging system: 1-D, 2-D and 3-D imaging of troposphere and stratosphere, Radio Sci., 43, RS2013, doi:10.1029/2006RS003603, 2008.

Isom, B., Palmer, R. D., Kelley, D., Meier, J., Bodine, D., Yeary, M., Cheong, B.-L., Zhang, Y., Yu, T.-Y., and Biggerstaff, M. I.: The atmospheric imaging radar: simultaneous volumetric observations using a phased array weather radar, J. Atmos. Ocean. Tech., 30, 655-675, 2013.

Latteck, R., Singer, W., Rapp, M., Vandepeer, B., Renkwitz, T., Zecha, M., and Stober, G.: MAARSY: The new MST radar on And'øya: System description and first results, Radio Sci., 47, RS1006, doi:10.1029/2011RS004775, 2012.

Luce, H., Yamamoto, M., Fukao, S., Hélal, D., and Crochet, M.: A frequency domain radar interferometric imaging (FII) technique based on high-resolution methods, J. Atmos. Sol.-Terr. Phys., 63, 221-234, 2001.

Luce, H., Hassenpflug, G., Yamamoto, M., Fukao, S., and Sato, K.: High-resolution observations with MU radar of a $\mathrm{KH}$ instability triggered by an inertia-gravity wave in the upper part of a jet stream, J. Atmos. Sci., 65, 1711-1718, 2008.

Palmer, R. D., Gopalam, S., Yu, T.-Y., and Fukao, S.: Coherent radar imaging using Capon's method, Radio Sci., 33, 1585-1598, doi:10.1029/98RS02200, 1998.

Palmer, R. D., Yu, T.-Y., and Chilson, P. B.: Range imaging using frequency diversity, Radio Sci., 34, 1485-1496, doi:10.1029/1999RS900089, 1999.

Palmer, R. D., Cheong, B. L., Hoffman, M. W., Fraser, S. J., and López-Dekker, F. J.: Observations of the small-scale variability of precipitation using an imaging radar, J. Atmos. Ocean. Tech., 22, 1122-1137, 2006.

Sureshbabu, V. N., Anandan, V. K., Tsuda, T., Furumoto, J., and Rao, S. V.: Performance analysis of optimum tilt angle and beam configuration to derive horizontal wind velocities by postset beam steering technique, IEEE T. Geosci. Remote, 51, 520526, doi:10.1109/TGRS.2012.2200256, 2013.

Woodman, R. F.: Coherent radar imaging: Signal processing and statistical properties, Radio Sci., 32, 2372-2391, doi:10.1029/97RS02017, 1997.

Yu, T.-Y. and Palmer, R. D.: Atmospheric radar imaging using multiple-receiver and multiple-frequency techniques, Radio Sci., 36, 1493-1503, doi:10.1029/2000RS002622, 2001.

Yu, T.-Y., Palmer, R. D., and Chilson, P. B.: An investigation of scattering mechanisms and dynamics in PMSE using coherent radar imaging, J. Atmos. Sol.-Terr. Phys., 63, 1797-1810, 2001.

Yu, T.-Y., Furumoto, J., and Yamamoto, M.: Clutter suppression for high-resolution atmospheric observations using multiple receivers and multiple frequencies, Radio Sci., 45, RS4011, doi:10.1029/2009RS004330, 2010. 